\title{
Allergy to dental amalgam
}

\author{
Miglena Balcheva ${ }^{1}$, Vladimir Panov ${ }^{1}$, \\ Assya Krasteva $^{2}$, Martina Markova ${ }^{1}$
}
1. Department of Conservative Dentistry and Oral Pathology, Faculty of dental medicine, Medical University -Varna;

2. Department of Oral Imaging and Oral Diagnostics, Faculty of dental medicine, Medical University -Sofia;

\begin{abstract}
Amalgam has been used for the restoration of teeth for more than 200 years, and it is may be the most successful of the direct restorative materials. One of the principal disadvantages of amalgam is that it may have adverse biological effects, both locally and systemically. It can cause an erythematous lesion on the adjacent oral soft tissues locally, and free mercury in the amalgam may give rise to a hypersensitivity reaction systemically.
\end{abstract}

The rare cases of allergic reactions in about $4 \%$ of tested patients we explain with the large oral tolerance and adaptive abilities of the oral cavity. We do not detect allergies in any of the dental medicine doctors. There is no positive test for both mercury and general product. Work with dental amalgam does not contribute to allergy as much as the persistent presence of the metal in the oral cavity. We may presume that dentists, being in constant contact with dental amalgam develop a kind of specific hyposensitization.

Keywords: dental amalgam, allergy, skin allergy tests

\section{Introduction}

Amalgam has been used for the restoration of teeth for more than 200 years, and it is maybe the most successful of the direct restorative materials. Despite the increasing use of tooth-coloured materials, with advantages of aesthetics and adhesion, amalgam is one of the most widely used dental restorative 
materials. One of the principal disadvantages of amalgam, apart from aesthetics, is that it may have adverse biological effects, both locally and systemically. It can cause an erythematous lesion on the adjacent oral soft tissues locally (tongue and buccal mucosa), and free mercury in the amalgam may give rise to hypersensitivity reaction systemically (McCullough MJ, 2008). Mercury is considered the main sensitiser in the amalgam, and contact with mercury salts such as chloride or ammonium chloride may cause sensitization, resulting in contact dermatitis (Coomb's Type IV hypersensitivity reactions). This is well known to the medical community (Fujii Y, 2017). Other typical manifestations are erythematous or urticarial rash, and pruritus on the face and flexural aspects of limbs. True allergy to mercury is rare, and it is more common in females (Bains VK, 2008).

Allergy may affect both the dental personnel and the patient. It may manifest intraorally as a lichenoid reaction or extraorally. The condition is rare and should not be confused with mercury toxicity. Unnecessary removal of sound amalgam restorations in healthy patients is not justified $\neg$ (Bains $R, 2012$; Panov Vl, 2016).

Exposure to mercury vapour, produced in dental premises, is the main concern for dentists. Therefore, all the possible precautions should be taken to avoid contact with mercury vapour. Recommendations include use of barrier techniques, air conditioning, lower temperature and proper ventilation of the dental premises, good suction, use of coolant sprays, proper handling of amalgam waste. Though amalgam is capable of producing delayed hypersensitivity reactions in some individuals, if the recommended precautions are followed, the risks of adverse health effects could be minimized (Rathore M, 2012).

Oral lichenoid lesions (OLL) occur more often as adverse reactions to dental amalgam than to other materials, present in patients' mouths. Oral lichenoid lesions caused by contact with dental amalgam are difficult to distinguish clinically or histologically from other lichenoid lesions or from idiopathic oral lichen planus (Panov Vl, 2016). Patients with OLL of unclear etiology, adjacent to large amalgam restorations, should be investigated for delayed contact hypersensitivity (Lynch M, 2015).

Various reports show the use of MELISA (memory lymphocyte immunostimulation assay) and patch tests in determining mercury sensitization (Bains VK, 2008). In cases of positive patch test results to mercury compounds, the replacement of amalgam fillings may lead to a significant improvement in most patients (Laeijendecker R, 2004; Lynch M, 2015; Panov VI, 2016).

Generally, WHO determines dental amalgam as relatively safe for restoring carious teeth, as it rarely causes local or allergic reactions. The small amount of mercury emitted during the placement or removal of amalgam filling does not affect human health (Panov VI, 2016). But, there is a rise in the allergic reactions to different materials in the the last few years, so dental practitioners should be aware about the documented cases and try to avoid such manifestations in their practices (Syed M, 2015).

\section{Aim}

The aim of our research is to establish the incidence of allergy to dental amalgam among patients and dentists. 


\section{Material and methods}

We study 2 groups of people. First group consists of 116 patients who have visited Faculty of Dental Medicine at the Medical University of Varna in the period 2014-2018. All of them have various complaints cheilitis, lip swelling, xerostomia, lichen planus, glossitis, stomatitis, rhinitis, psoriasis, angioedema, metallic taste, mental problems, burning mouth syndrome, and all of them are or have been exposed to dental amalgam. The average age of these patients is 45.61 years, Rank (8-82), standard deviation 18.28. Twentyfour of them are men and 92 women.

Second group, investigated in October 2018, consists of 20 dental practitioners without complaints.

We use patch testing as a diagnostic tool - classic method for contact hypersensitivity determination, proposed and described by J. Jadassohn in 1895 and further developed by Bloch in 1910. The only requirement for the test subjects is to discontinue anti-allergic drugs (antihistamines, corticosteroids, etc.) at least 5 days before and during the study.

We use standard mercury allergen, hypoallergenic patches with polyethylene chambers and skin marker from Chemotechnique Diagnostics (Vellinge, Sweden), and amalgam-general product allergen prepared at NCIPD-Sofia, Laboratory of Allergies.

In the first group, patches are applied on skin of the back (healthy, glabrous skin without tanning) in the area between the first thoracic vertebra, crista iliaca and the spine, and results are read at the 48-th hour. In the second group patches are applied on the skin of the forearm, results are read on the 24-th hour, and sensitization to mercury and amalgam-general product is compared.

Results are read according to the standard adopted by the International Contact Dermatitis Research Group (ICDRG):

- (+?) - doubtful reaction - faint homogenous erythema

- $(+)$ - weak positive reaction - erythema, papules, infiltration

- $\quad(++)$ - strong positive reaction - erythema, papules, infiltration, discrete vesicles

- $(+++)$ - extreme positive reaction - marked erythema, infiltration, coalescing vesicles, bullous or ulcerative reaction

- $(-)$ - negative reaction

- (IR) - irritant reaction - discrete patchy erythema without infiltration that subsides soon after patch removal

\section{Results}

Negative test results (Value 0) prevail in the studied group (Fig.1). Positive results are observed just in 4\% of cases, as $3 \%$ are weak positive reactions (Value 1), $1 \%$ are strong positive reactions (Value 2), and there are no extreme positive reactions (Value 3) (Fig.2). 
All the positive results are read in the first studied group. These are five patients, which are respectively 21 , 22, 41, 52 and 57 years old. The average age of the positives patients is 38.9 years, rank (21-57) and standard deviation 16.65. All of them are females.

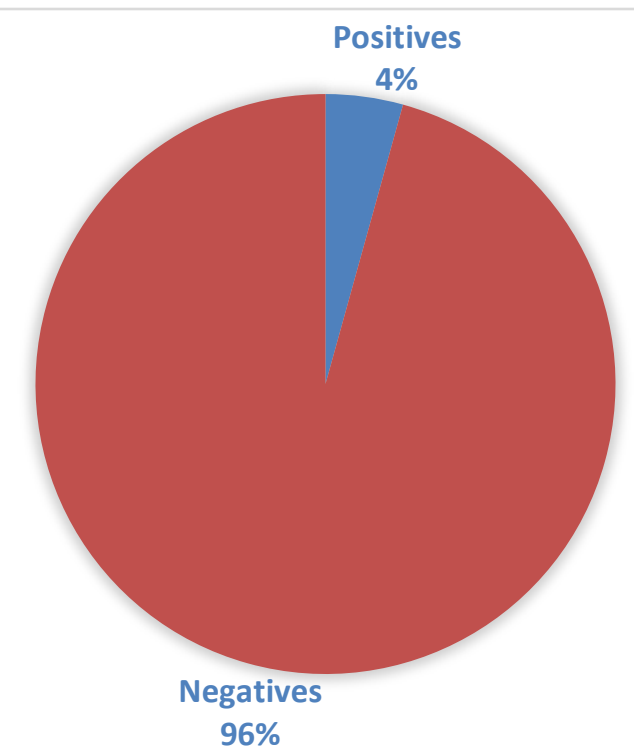

Figure 1. Patch test results

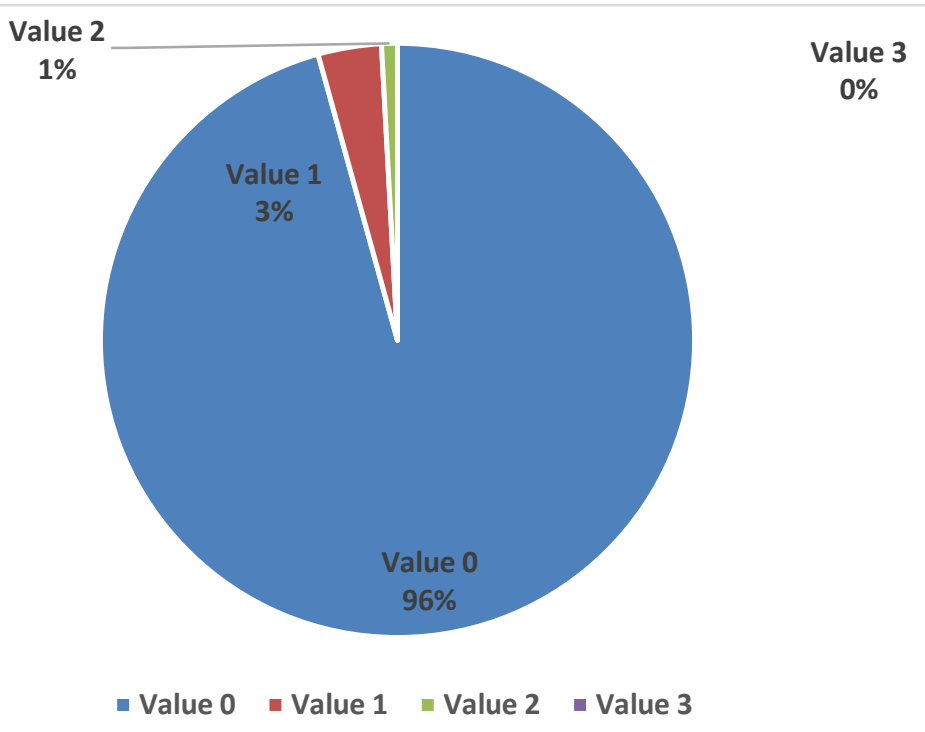

Figure 2. Share distribution of the different patch test results 
It is noteworthy that positive reactions to dental amalgam are observed relatively rarely (Fig.3). And these positive reactions are usually weak (Fig.4).

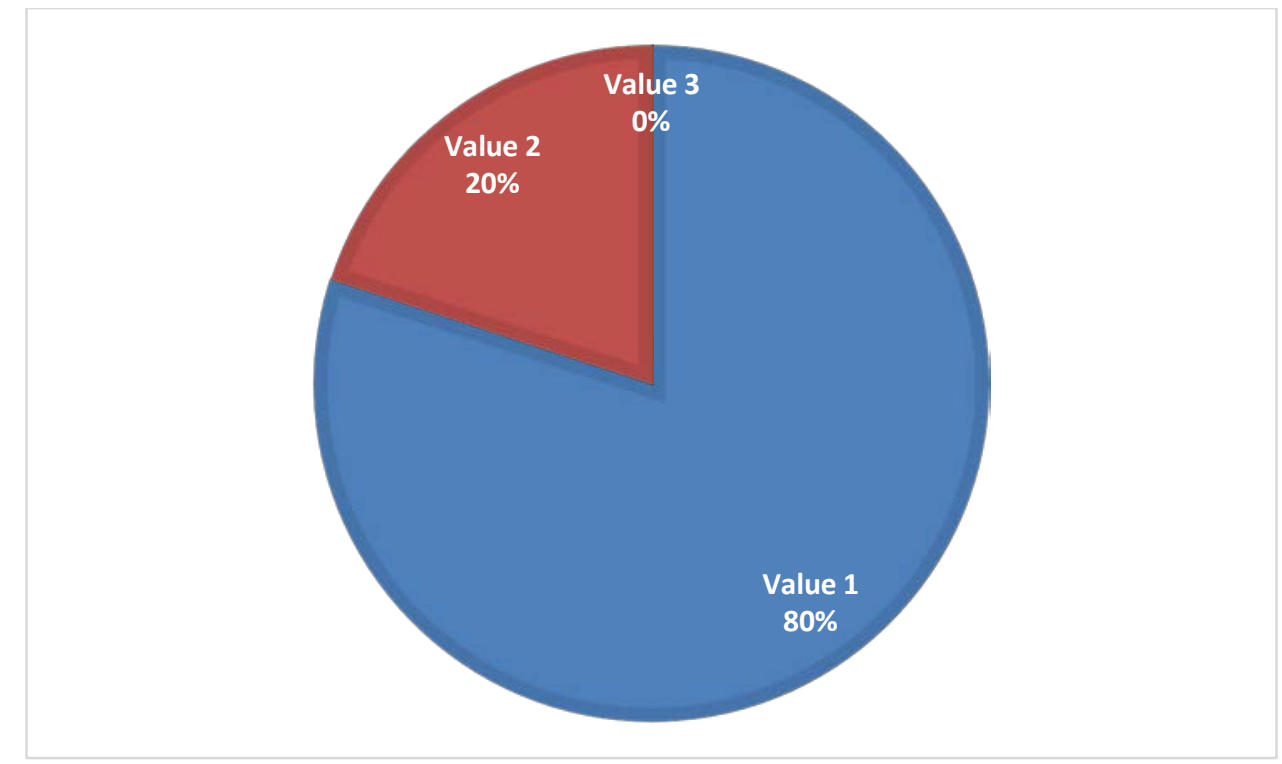

Figure 3. Share distribution of the positive patch test results

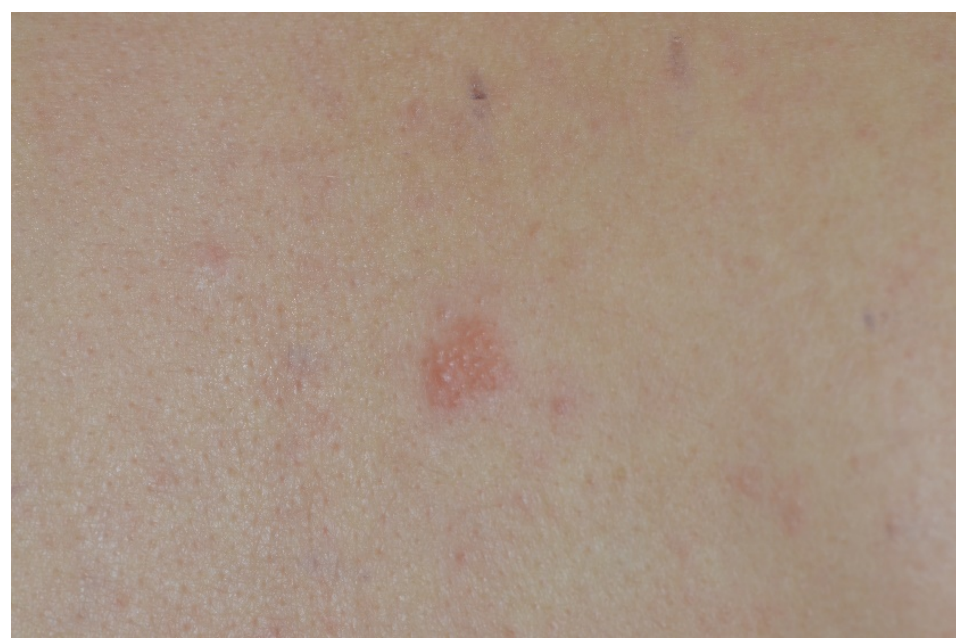

Figure 4. Positive patch test results 


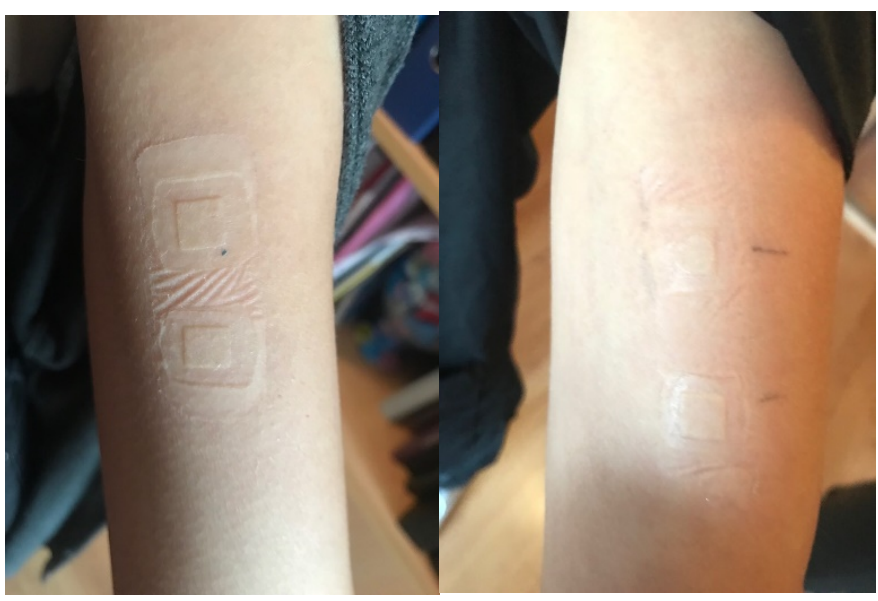

Figure 5. Negative patch test results

No allergy is detected in the group of dental practitioners. There is no positive test for both mercury and amalgam-general product (Fig.5).

\section{Discussion}

Taking into account our previous studies of the presence of dental amalgam in dental practitioners (16\%), and in the total population (48\%), we can conclude, with the limitation of this study that allergy to dental amalgam is very rare and affects about $2 \%$ of the population (Markova $M, 2016$ ). Low frequency of allergic reactions (4\%) is explained by the large oral tolerance and adaptive abilities of the oral cavity. Working with dental amalgam does not contribute to allergy as much as the persistent presence of the metal in the oral cavity. Moreover, dental practitioners, being in constant contact with dental amalgam, maybe develop a kind of specific hyposensitization.

\section{References}

1. Bains VK, Loomba K, Loomba A, Bains R, Mercury sensitisation: review, relevance and a clinical report. Br Dent J. 2008 Oct 11;205(7):373-378.

2. Laeijendecker R, Dekker SK, Burger PM, Mulder PG, Van Joost T, Neumann MH. Oral lichen planus and allergy to dental amalgam restorations. Arch Dermatol. 2004 Dec;140(12):1434-1438.

3. Lynch M, Ryan A, Galvin S, Flint S, Healy CM, O'Rourke N, Lynch K, Rogers S, Collins P. Patch testing in oral lichenoid lesions of uncertain etiology. Dermatitis. 2015 Mar-Apr;26(2):89-93.

4. Markova M, Panov VI, Georgiev G, Vicheva G, Dental amalgam -Clinical-epidemiological aspects, Varna Medical Forum, p. 5, 2016, issue 2, 210-213.

5. McCullough MJ, Tyas MJ, Local adverse effects of amalgam restorations, Int Dent J. 2008 Feb;58(1):3-9.

6. Panov $\mathrm{Vl}$, Adverse reactions in the mouth when using dental amalgam, Varna Medical Forum, 2016, 5(1), 77-81. 
7. Panov Vl, M. Lyapina, M. Markova, Recommendations for clinical practice in the use of dental amalgam, in Biocompatibility in Oral Medicine, Medinform, 2016, 229-234.

8. Panov Vl, Reactions in the oral cavity due to Dental Amalgam, Biocompatibility in Oral Medicine, Medinform, 2016, 225-228.

9. Panov Vl., M. Markova, Historical Review and Toxicological Characteristics of Dental Amalgam, in Biocompatibility in Oral Medicine, Medinform, 2016, 200-209.

10. Rathore M, Singh A, Pant VA, The dental amalgam toxicity fear: a myth or actuality, Toxicol Int. 2012 May;19(2):81-88.

11. Rhythm Bains1, Kapil Loomba1 and Anju Loomba, Allergy to Mercury from Dental Amalgam: A Case Report, Asian Journal of Oral Health \& Allied Sciences 2012, Volume 2, Issue 2, 91-93.

12. Syed M, Chopra R, Sachdev V, Allergic Reactions to Dental Materials-A Systematic Review, J Clin Diagn Res. 2015 Oct; 9(10): ZE04-ZE09.

\section{Corresponding author:}

Vladimir Panov, Faculty of Dental Medicine,

Department of Conservative Dentistry and Oral Pathology, Medical University - Varna, email: vladimir.panov@mu-varna.bg 